\title{
Credit Risk Evaluation of Big Data Enterprises Based on Intuitionistic Fuzzy Sets
}

\author{
Mu Zhang ${ }^{1, *} \mathrm{Si}$-si Li ${ }^{1,2}$ \\ ${ }^{1}$ School of Big Data Application and Economics, Guizhou University of Finance and Economics \\ ${ }^{2}$ Guizhou Institution for Technology Innovation \& Entrepreneurship Investment, Guizhou University of Finance and \\ Economics \\ ${ }^{*}$ Corresponding author. Email: rim_007@163.com
}

\begin{abstract}
The fuzzy sets theory cannot accurately reflect the hesitation or abstention of decision makers. In view of this problem, introducing the intuitionistic fuzzy sets, we put forward a credit risk evaluation model of big data enterprises based on intuitionistic fuzzy sets. Firstly, a credit risk evaluation index system of big data enterprises was constructed. Meanwhile, the intuitionistic fuzzy weights of indicators were determined with intuitionistic fuzzy AHP. Secondly, we constructed the IFWA operator, and then the intuitionistic fuzzy comprehensive evaluation value of sample enterprise was calculated. Finally, we used the score function of intuitionistic fuzzy number to calculate the credit comprehensive score and ranking of sample enterprise. Taking three listed companies in big data industry in China as sample, we used the above model to carry out an example analysis. The result of example analysis proves the feasibility and effectiveness of this model.
\end{abstract}

Keywords: big data enterprises, credit risk, credit risk evaluation, intuitionistic fuzzy sets, intuitionistic fuzzy number

\section{基于直觉模糊集的大数据企业信用风险评价研究}

\author{
张 目 ${ }^{1, *}$ 李思思 ${ }^{1,2}$
}

大数据应用与经济学院, 贵州财经大学

2 贵州科技创新创业投资研究院，贵州财经大学

*通讯作者. 电子邮箱: rim 007@163. com

\section{摘要}

文章针对传统模糊集理论不能准确反映决策者犹豫不决或弃权情况的问题，引入直觉模糊集，构建基于直觉 模糊集的大数据企业信用风险评价模型。首先，构建大数据企业信用风险评价指标体系，并采用直觉模糊层 次分析法确定指标的直觉模糊权重; 然后，构造直觉模糊加权平均算子，计算待评企业的直觉模糊综合评价 值; 最后，利用直觉模糊数记分函数，计算得出待评企业的信用综合记分和排序。以大数据产业上市公司为 样本，实例分析结果证明了该模型的可行性和有效性。

关键字: 大数据企业, 信用风险, 信用风险评价, 直觉模糊集, 直觉模糊数.

\section{1. 引言}

大数据产业指以数据生产、采集、存储、加工、 分析、服务为主的相关经济活动, 包括数据资源建 设、大数据软硬件产品的开发、销售和租赁活动,
以及相关信息技术服务。抢抓机遇, 推动大数据产 业发展，对提升政府治理能力、优化民生公共服务、 促进经济转型和创新发展有重大意义 ${ }^{[1]}$ 。如何科学 评价大数据企业信用风险, 是 “鼓励金融机构加强 和改进金融服务，加大对大数据企业的支持力度” [2] 
的基础性和关键性课题。企业信用风险评价问题可 视为一个不确定多属性决策问题, 其中, 由于决策 者主观思维的模糊性、不确定性和信用风险的复杂 多变性, 决策者对信用风险属性的评判往往具有模 糊性，因而模糊综合评判法等基于模糊集理论的多 属性决策方法被逐渐引入到企业信用风险评价中 ${ }^{[3-4]}$ 然而, 传统模糊集理论不能准确反映决策者犹豫不 决或弃权的情况。为此, Atanassov ${ }^{[5]}$ 对 $Z^{2} \operatorname{coh}^{[6]}$ 的模 糊集理论进行了推广, 提出了直觉模糊集的概念, 能够较好、较完整地反映决策主体对客观事物评价 的模糊性和不确定性, 已成功应用于不确定多属性 决策领域 $[7-8]$ 。有鉴于此, 本文尝试将直觉模糊集引 入到大数据企业信用风险评价中, 具体结构安排如 下：第一，构建大数据企业信用风险评价指标体系; 第二, 介绍直觉模糊集及相关定义; 第三, 采用直 觉模糊层次分析法确定各级指标的直觉模糊权重, 并引入直觉模糊加权平均算子和直觉模糊数记分函 数, 构建基于直觉模糊集的大数据企业信用风险评 价模型; 最后, 以大数据产业上市公司为样本, 运 用该模型进行实例分析。

\section{2. 大数据企业信用风险评价指标体系构建}

\section{表 1 大数据企业信用风险评价指标体系}

\begin{tabular}{|l|l|}
\hline \multicolumn{1}{|c|}{ 一级指标 } & \multicolumn{1}{|c|}{ 二级指标 } \\
\hline 偿债能力 & $\begin{array}{l}\text { 流动比率 } U_{11} \text {; 速动比率 } U_{12} \text {; 资产 } \\
U_{1}\end{array}$ 负债率 $U_{13}$ \\
\hline 营运能力 & 应收账款周转率 $U_{21}$; 总资产周转率 \\
$U_{2}$ & $U_{22}$; 存货周转率 $U_{23}$ \\
\hline 盈利能力 & 主营业务利润率 $U_{31}$; 净资产收益率 \\
$U_{3}$ & $U_{32}$; 总资产收益率 $U_{33}$ \\
\hline 成长能力 & 净利润增长率 $U_{41}$; 主营业务增长率 \\
$U_{4}$ & $U_{42}$ \\
\hline 技术创新 & 开发支出 $U_{51}$; 无形资产增长率 \\
能力 $U_{5}$ & $U_{52}$; 发明专利申请公布数 $U_{53}$ \\
\hline 行业成长 & 行业平均净利润增长率 $U_{61}$; 行业平 \\
性 $U_{6}$ & 均营业收入增长率 $U_{62}$ \\
\hline
\end{tabular}

企业信用风险是指在信贷过程中, 由于各种不 确定性, 使借款企业不能按时偿还贷款, 造成银行 贷款本金及利息损失的可能性。本文根据企业信用 风险的内涵, 综合国内外相关研究成果, 参照中国 人民银行信用评级管理指导意见（银发〔2006)95 号）和科技型中小企业信用评估基本要素（如基础 素质、商业经营、财务状况、创新能力、外部环境 等) [9], 结合大数据企业特征（如技术创新能力、 行业成长性等), 遵循科学性、客观性、系统性、 功能性、动态性、相对独立性、可行性（或可操作 性）和可比性等评价指标的选取原则，从偿债能力、 营运能力、盈利能力、成长能力、技术创新能力、 行业成长性等几个方面构建了包含 6 个一级指标和
16 个二级指标的大数据企业信用风险评价指标体系, 如表 1 所示。

\section{3. 直觉模糊集及相关定义}

定义 $1^{[5]}$ 设 $X$ 是一个非空集合，则称 $A=\left\{\left\langle x, \mu_{A}(x), v_{A}(x)\right\rangle \mid x \in X\right\}$ 为直觉模糊集, 其中 $\mu_{A}(x)$ 和 $\nu_{A}(x)$ 分别为 $X$ 中元素 $x$ 属于 $A$ 的隶属度和 非隶属度, 即有 $\mu_{A}: X \rightarrow[0,1], v_{A}: X \rightarrow[0,1]$, 且 满足 $0 \leq \mu_{A}(x)+v_{A}(x) \leq 1, \forall x \in X$ 。同 时 称 $\pi_{A}(x)=1-\mu_{A}(x)-v_{A}(x)$ 为 $X$ 中元素 $x$ 属于 $A$ 的犹豫 度, 且满足 $0 \leq \pi_{A}(x) \leq 1, \forall x \in X$ 。为方便起见, 设 $\alpha=\left(\mu_{\alpha}, v_{\alpha}\right)$ 为直觉模糊数, 其中 $\mu_{\alpha} \in[0,1]$, $v_{\alpha} \in[0,1], 0 \leq \mu_{\alpha}+v_{\alpha} \leq 1$, 并设 $\Theta$ 为全体直觉模糊 数的集合。

定义 $2^{[5]}$ 设 $a_{1}=\left(\mu_{1}, v_{1}\right)$ 和 $a_{2}=\left(\mu_{2}, v_{2}\right)$ 是给定论 域上的直觉模糊数; 设 $\lambda$ 为实数, 且 $\lambda>0$, 则直觉 模糊数的运算法则为:

1) $a_{1} \oplus a_{2}=\left(\mu_{1}+\mu_{2}-\mu_{1} \mu_{2}, v_{1} v_{2}\right)$;

2) $a_{1} \otimes a_{2}=\left(\mu_{1} \mu_{2}, v_{1}+v_{2}-v_{1} v_{2}\right)$;

3) $\lambda a_{1}=\left(1-\left(1-\mu_{1}\right)^{\lambda}, v_{1}^{\lambda}\right)$;

4) $a_{1}^{\lambda}=\left(\mu_{1}^{\lambda}, 1-\left(1-v_{1}\right)^{\lambda}\right)$ 。

定义 3 设 $\alpha_{j}=\left(\mu_{\alpha_{j}}, v_{\alpha_{j}}\right)(j=1,2, \cdots, n)$ 为一组直 觉模糊数, $\tilde{w}_{j}=\left(\mu_{\tilde{w}_{j}}, v_{\tilde{w}_{j}}\right)(j=1,2, \cdots, n)$ 为 $\alpha_{j}$ 的直觉 模 糊 权 重 , $\mu_{\tilde{w}_{j}} \in[0,1] ， v_{\tilde{w}_{j}} \in[0,1]$ ，且 $\mu_{\tilde{w}_{j}}+v_{\tilde{w}_{j}} \in[0,1]$ ，则由 IFWA 算子得到的集成值也是 直觉模糊数。且设 IFWA : $\Theta^{n} \rightarrow \Theta$, 若

$\operatorname{IFWA}_{\tilde{w}}\left(\alpha_{1}, \alpha_{2}, \cdots, \alpha_{n}\right)=\tilde{w}_{1} \otimes \alpha_{1} \oplus \tilde{w}_{2} \otimes \alpha_{2} \oplus \cdots \oplus \tilde{w}_{n} \otimes \alpha_{n}$

则称 IFWA 为直觉模糊加权平均算子。

当 $\tilde{w}_{j}$ 退化为经典权重 $w_{j}$ 时，即 $w_{j} \in[0,1]$, 且

$\sum_{j=1}^{n} w_{j}=1$, 则 IFWA 算子退化为 $[10]$

$$
\begin{aligned}
& \operatorname{IFWA}_{w}\left(\alpha_{1}, \alpha_{2}, \cdots, \alpha_{n}\right)=w_{1} \alpha_{1} \oplus w_{2} \alpha_{2} \oplus \cdots \oplus w_{n} \alpha_{n} \\
& =\left(1-\prod_{j=1}^{n}\left(1-\mu_{\alpha_{j}}\right)^{w_{j}}, \prod_{j=1}^{n} v_{\alpha_{j}}^{w_{j}}\right)
\end{aligned}
$$

定义 $4^{[11]}$ 设某一论域 $U_{j}$ 下有 $n$ 个直觉模糊数 $\alpha_{1 j}, \alpha_{2 j}, \cdots, \alpha_{n j}, \theta_{i j}=\operatorname{shi}\left(\alpha_{i j}\right) / \operatorname{shi}\left(I_{j}\right)$ 为直觉模糊数 $\alpha_{i j}$ 的相对集 对势, 其 中 $\alpha_{i j}=\left(\mu_{i j}, v_{i j}\right)$, $\operatorname{shi}\left(\alpha_{i j}\right)=\mu_{i j} / v_{i j}, \operatorname{shi}\left(I_{j}\right)=\sum_{i=1}^{n} \mu_{i j} / \sum_{i=1}^{n} v_{i j}$, 则 $\alpha_{i j}$ 的记 分函数为

$$
S\left(\alpha_{i j}\right)=\mu_{i j}-v_{i j}+\frac{\theta_{i j}-1}{\theta_{i j}+1} \pi_{i j}
$$

若 $S\left(\alpha_{1 j}\right)>S\left(\alpha_{2 j}\right)$, 则 $\alpha_{1 j}>\alpha_{2 j}$ 。 


\section{4. 大数据企业信用风险评价模型}

\section{1. 直觉模糊层次分析法}

步骤 1: 构建指标体系。由表 1 可知, 大数据企 业信用风险评价指标体系分为两个层次，即有：

$$
\begin{aligned}
& U=\left(U_{1}, U_{2}, \cdots, U_{i}, \cdots, U_{n}\right), \quad i=1,2, \cdots, n ; \\
& U_{i}=\left(U_{i 1}, U_{i 2}, \cdots, U_{i k}, \cdots, U_{i n_{i}}\right), \quad k=1,2, \cdots, n_{i} 。
\end{aligned}
$$

其中, $n$ 表示一级指标的个数; $n_{i}$ 表示第 $i$ 个一级指 标下二级指标的个数。

步骤 2: 确定专家权重。设有 $p$ 位专家, $b_{i j}$ 是专

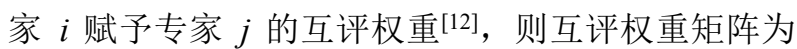
$B=\left(b_{i j}\right)_{p \times p}$, 其中 $0 \leq b_{i j} \leq 1$, 且 $\sum_{j=1}^{p} b_{i j}=1$, 则群 体赋予专家 $j$ 的权重为 $q_{j}=p^{-1} \sum_{i=1}^{p} b_{i j}$ 。由此, 通过 循环评价来确定专家的最终权重。设 $t$ 为互评次数, 当 $t=1$ 时, 专家权重向量为 $q^{1}=\left(q_{1}, q_{2}, \cdots, q_{p}\right)$ ；当 $t>1$ 时, 专家权重向量为 $q^{t}=q^{t-1} B$, 直至 $q^{t}$ 收玫于 稳定值。

\begin{tabular}{|c|c|c|}
\hline $\begin{array}{l}\text { 直觉模 } \\
\text { 糊标度 }\end{array}$ & 语意含义 & 直觉模糊数 \\
\hline$\tilde{1}$ & $\begin{array}{c}\text { 两因素相比, 因素 } i \\
\text { 比因素 } j \text { 极端重要 }\end{array}$ & $(0.90,0.10,0.00)$ \\
\hline$\tilde{2}$ & $\begin{array}{c}\text { 两因素相比, 因素 } i \\
\text { 比因素 } j \text { 重要得多 }\end{array}$ & $(0.80,0.15,0.05)$ \\
\hline$\tilde{3}$ & $\begin{array}{c}\text { 两因素相比, 因素 } i \\
\text { 比因素 } j \text { 明显重要 }\end{array}$ & $(0.70,0.20,0.10)$ \\
\hline$\tilde{4}$ & $\begin{array}{c}\text { 两因素相比, 因素 } i \\
\text { 比因素 } j \text { 稍微重要 }\end{array}$ & $(0.60,0.25,0.15)$ \\
\hline$\tilde{5}$ & $\begin{array}{c}\text { 两因素相比, 因素 } i \\
\text { 与因素 } j \text { 同等重要 }\end{array}$ & $(0.50,0.30,0.20)$ \\
\hline$\tilde{6}$ & $\begin{array}{c}\text { 两因素相比, 因素 } j \\
\text { 比因素 } i \text { 稍微重要 }\end{array}$ & $(0.40,0.45,0.15)$ \\
\hline$\tilde{7}$ & $\begin{array}{c}\text { 两因素相比, 因素 } j \\
\text { 比因素 } i \text { 明显重要 }\end{array}$ & $(0.30,0.60,0.10)$ \\
\hline$\tilde{8}$ & $\begin{array}{c}\text { 两因素相比, 因素 } j \\
\text { 比因素 } i \text { 重要得多 }\end{array}$ & $(0.20,0.75,0.05)$ \\
\hline$\tilde{9}$ & $\begin{array}{c}\text { 两因素相比, 因素 } j \\
\text { 比因素 } i \text { 极端重要 }\end{array}$ & $(0.10,0.90,0.00)$ \\
\hline
\end{tabular}

\section{表 2 直觉模糊标度的语意含义及直觉模糊数}

步骤 3: 构造直觉模糊判断矩阵。由 $p$ 位专家, 根据 $\tilde{1} \sim \tilde{9}$ 直觉模糊标度 ${ }^{[13]}$, 对一级指标的指标两 两重要性进行比较。根据专家权重, 由式（2）集结 $p$ 位专家意见, 从而构造直觉模糊判断矩阵 $R=\left(r_{i j}\right)_{n \times n}, i$ 和 $j$ 分别表示直觉模糊判断矩阵中的行 和列, 其中 $r_{i j}=\left(\mu_{i j}, v_{i j}\right), \mu_{i j}$ 表示隶属度, 即第 $i$ 个 指标优于第 $j$ 个指标的程度; $v_{i j}$ 表示非隶属度, 即 第 $i$ 个指标劣于第 $j$ 个指标的程度; $\pi_{i j}$ 表示犹豫度, $\pi_{i j}=1-\mu_{i j}-v_{i j}$ 。直觉模糊标度对应的语意含义及直 觉模糊数如表 2 所示。

步骤 4: 一致性检验。根据直觉模糊信息的距离 测度 ${ }^{[14],}$ 给出下列一致性检验公式 ${ }^{[15]}$ :

$d(\bar{R}, R)=\frac{1}{2(n-1)(n-2)} \sum_{i=1}^{n} \sum_{j=1}^{n}\left(\left|\bar{\mu}_{i j}-\mu_{i j}\right|+\left|\bar{v}_{i j}-v_{i j}\right|+\left|\bar{\pi}_{i j}-\pi_{i j}\right|\right)$

其中, $R$ 为直觉模糊判断矩阵, $\bar{R}$ 为由 $R$ 计算得到 的直觉模糊一致性判断矩阵 $\bar{R}=\left(\bar{r}_{i j}\right)_{n \times n}$, 其计算方法 如下:

1) 当 $j>i+1$ 时, 令 $\bar{r}_{i j}=\left(\bar{\mu}_{i j}, \bar{v}_{i j}\right)$, 其中 ${ }^{[14]}$

$$
\begin{gathered}
\bar{\mu}_{i j}=\frac{\sqrt[j-i-1]{\prod_{t=i+1}^{j-1} \mu_{i t} \mu_{t j}}}{\sqrt[j-i-1]{\prod_{t=i+1}^{j-1} \mu_{i t} \mu_{t j}}+\sqrt[j-i-1]{\prod_{t=i+1}^{j-1}\left(1-\mu_{i t}\right)\left(1-\mu_{t j}\right)}} \\
\bar{v}_{i j}=\frac{\sqrt[j-i-1]{\prod_{t=i+1}^{j-1} v_{i t} v_{t j}}}{\sqrt[j-i-1]{\prod_{t=i+1}^{j-1} v_{i t} v_{t j}}+\sqrt[j-i-1]{\prod_{t=i+1}^{j-1}\left(1-v_{i t}\right)\left(1-v_{t j}\right)}}
\end{gathered}
$$

2) 当 $j=i$ 或 $j=i+1$ 时, 令 $\bar{r}_{i j}=\left(\mu_{i j}, v_{i j}\right)$;

$3)$ 当 $j<i$ 时, 令 $\bar{r}_{i j}=\left(\bar{v}_{i j}, \bar{\mu}_{i j}\right)$ 。

将计算得出的 $\bar{R}$ 代入式 (4) 进行一致性检验。 若 $d(\bar{R}, R)<0.1$, 则通过一致性检验, 否则未通过一 致性检验。

步骤 5: 调整未通过一致性检验的直觉模糊一致 性判断矩阵。设置迭代参数 $\sigma(\sigma \in[0,1])$ 对直觉 模糊一致性判断矩阵 $\bar{R}$ 进行调整。令 ${ }^{[15]}$

$$
\begin{aligned}
& \tilde{\mu}_{i j}=\frac{\left(\mu_{i j}\right)^{1-\sigma}\left(\bar{\mu}_{i j}\right)^{\sigma}}{\left(\mu_{i j}\right)^{1-\sigma}\left(\bar{\mu}_{i j}\right)^{\sigma}+\left(1-\mu_{i j}\right)^{1-\sigma}\left(1-\bar{\mu}_{i j}\right)^{\sigma}} \\
& \tilde{v}_{i j}=\frac{\left(v_{i j}\right)^{1-\sigma}\left(\bar{v}_{i j}\right)^{\sigma}}{\left(v_{i j}\right)^{1-\sigma}\left(\bar{v}_{i j}\right)^{\sigma}+\left(1-v_{i j}\right)^{1-\sigma}\left(1-\bar{v}_{i j}\right)^{\sigma}}
\end{aligned}
$$

由此可得调整后的直觉模糊一致性判断矩阵 $\tilde{R}=\left(\tilde{r}_{i j}\right)_{n \times n}$, 其中 $\tilde{r}_{i j}=\left(\tilde{\mu}_{i j}, \tilde{v}_{i j}\right)$ 。将 $\tilde{R}$ 代入下式进行 一致性检验，直至 $\tilde{R}$ 通过一致性检验。

$d(\tilde{R}, R)=\frac{1}{2(n-1)(n-2)} \sum_{i=1}^{n} \sum_{j=1}^{n}\left(\left|\tilde{\mu}_{i j}-\mu_{i j}\right|+\left|\tilde{v}_{i j}-v_{i j}\right|+\left|\tilde{\pi}_{i j}-\pi_{i j}\right|\right)$

步骤 6: 计算指标的直觉模糊权重。依据通过一 致性检验的直觉模糊一致性判断矩阵, 一级指标的 直觉模糊权重为 ${ }^{[15]}$ :

$$
\tilde{w}_{i}=\left(\frac{\sum_{j=1}^{n} \tilde{\mu}_{i j}}{\sum_{i=1}^{n} \sum_{j=1}^{n}\left(1-\tilde{v}_{i j}\right)}, 1-\frac{\sum_{j=1}^{n}\left(1-\tilde{v}_{i j}\right)}{\sum_{i=1}^{n} \sum_{j=1}^{n} \tilde{\mu}_{i j}}\right)
$$


则一级指标的直觉模糊权重向量为

$$
\tilde{W}=\left(\tilde{w}_{1}, \tilde{w}_{2}, \cdots, \tilde{w}_{i}, \cdots, \tilde{w}_{n}\right) 。
$$

同理可得二级指标的直觉模糊权重向量:

$$
\tilde{W}_{i}=\left(\tilde{w}_{i 1}, \tilde{w}_{i 2}, \cdots, \tilde{w}_{i k}, \cdots, \tilde{w}_{i n_{i}}\right) 。
$$

\section{2. 指标评价语意变量与直觉模糊数}

语意变量是以人类的自然语言中的语词为值。 考虑到指标评价的模糊性和主观性, 本文的指标评 价采用语意变量来表示。指标评价语意变量取 7 个 等级: 非常差、差、较差、一般、较好、好、非常 好。指标评价语意变量与直觉模糊数的对应关系如 表 3 所示。

\section{表 3 指标评价语意变量与直觉模糊数的对应关系}

\begin{tabular}{|c|c|}
\hline 指标评价语意变量 & 直觉模糊数 \\
\hline 非常差 & $(0.05,0.95,0.00)$ \\
\hline 差 & $(0.20,0.75,0.05)$ \\
\hline 较差 & $(0.35,0.50,0.15)$ \\
\hline 一般 & $(0.50,0.30,0.20)$ \\
\hline 较好 & $(0.65,0.20,0.15)$ \\
\hline 好 & $(0.80,0.15,0.05)$ \\
\hline 非常好 & $(0.95,0.05,0.00)$ \\
\hline
\end{tabular}

\section{3. 大数据企业信用风险评价步骤}

设有待评价大数据企业 $E_{j}(j=1,2, \cdots, m)$, 同 时考虑 $n$ 个一级指标, 且每个一级指标下有若干个 二级指标, 则大数据企业信用风险评价的具体步骤 为:

步骤 1: 根据 $p$ 位专家意见, 由 4.1 节, 计算得 出一级指标的直觉模糊权重向量 $\tilde{W}$ 和二级指标的直 觉模糊权重向量 $\tilde{W}_{i}$ 。

步骤 2: 针对待评价大数据企业 $E_{j}$ 在各一级指标 下的每个二级指标值, 由 $p$ 位专家根据表 3 分别给出 指标评价语意变量。根据专家权重, 由式 (2) 集结 $p$ 位专家意见, 得出待评价大数据企业 $E_{j}$ 在第 $i$ 个一 级指标下第 $k$ 个二级指标的直觉模糊评价值: $\tilde{x}_{j i k}=\left(\mu_{\tilde{x}_{j k}}, v_{\tilde{x}_{j i k}}\right)$ 。

步骤 3: 由式 (1) 可得待评价大数据企业 $E_{j}$ 在 第 $i$ 个一级指标下的直觉模糊评价值:

$\tilde{x}_{j i}=\tilde{x}_{j i 1} \otimes \tilde{w}_{i 1} \oplus \tilde{x}_{j i 2} \otimes \tilde{w}_{i 2} \oplus \cdots \oplus \tilde{x}_{j i k} \otimes \tilde{w}_{i k} \oplus \cdots \oplus \tilde{x}_{j n_{i}} \otimes \tilde{w}_{i n_{i}}$

步骤 4: 由式 (11) 和式 (1) 可得待评价大数 据企业 $E_{j}$ 的直觉模糊综合评价值:

$\tilde{x}_{j}=\tilde{x}_{j 1} \otimes \tilde{w}_{1} \oplus \tilde{x}_{j 2} \otimes \tilde{w}_{2} \oplus \cdots \oplus \tilde{x}_{j i} \otimes \tilde{w}_{i} \oplus \cdots \oplus \tilde{x}_{j n} \otimes \tilde{w}_{n}$

其中, $\tilde{x}_{j}=\left(\mu_{\tilde{x}_{j}}, v_{\tilde{x}_{j}}\right)$ 。

步骤 5: 由式 (3) 可得待评价大数据企业 $E_{j}$ 的 信用综合记分：

$$
S\left(\tilde{x}_{j}\right)=\mu_{\tilde{x}_{j}}-v_{\tilde{x}_{j}}+\frac{\theta_{j}-1}{\theta_{j}+1} \pi_{\tilde{x}_{j}}
$$

其中, $\theta_{j}=\operatorname{shi}\left(\tilde{x}_{j}\right) / \operatorname{shi}(I), \quad \operatorname{shi}\left(\tilde{x}_{j}\right)=\mu_{\tilde{x}_{j}} / v_{\tilde{x}_{j}}$, $\operatorname{shi}(I)=\sum_{j=1}^{m} \mu_{\tilde{x}_{j}} / \sum_{j=1}^{m} v_{\tilde{x}_{j}} 。$

若 $S\left(\tilde{x}_{1}\right)>S\left(\tilde{x}_{2}\right)$, 则 $\tilde{x}_{1}>\tilde{x}_{2}$, 表明待评价大数 据企业 $E_{1}$ 的信用状况优于 $E_{2}$, 其信用风险低于 $E_{2}$ 。

\section{5. 实例分析}

\section{1. 样本数据}

本文选取创业板中的大数据产业上市公司一一 网宿科技（300017）、立思辰 (300010)、天喻信息 (300205) 组成实验样本集 $\{A, B, C\}$ 。样本企业 $A$ 和 $B$ 所属证监会行业为: 信息传输、软件和信息技术 服务业-软件和信息技术服务业; 样本企业 $C$ 所属证 监会行业为: 制造业-计算机、通信和其他电子设备 制造业。样本区间设定为 2016 年。发明专利申请公 布数指标原始数据来源于天眼查网站, 其余指标原 始数据均来源于东方财富网站。限于篇幅, 原始数 据略。

\section{2. 大数据企业信用风险评价}

（1）确定专家权重

设有 3 位专家, 由 4.1 节步骤 2 , 互评权重矩阵 为

$$
B=\left[\begin{array}{lll}
0.50 & 0.25 & 0.25 \\
0.38 & 0.31 & 0.31 \\
0.28 & 0.36 & 0.36
\end{array}\right]
$$

当 $t=1$ 时, 专家权重 向量为 $q^{1}=(0.3866,0.3067,0.3067)$ ；当 $t=5$ 时，专家权重向 量收玫于稳定值 $q^{5}=(0.3976,0.3012,0.3012)$ 。

(2) 确定指标的直觉模糊权重

由 3 位专家, 根据 $\tilde{1} \sim \tilde{9}$ 直觉模糊标度 ${ }^{[13]}$, 对一 级指标的指标两两重要性进行比较。根据专家权重 $(0.3976,0.3012,0.3012)$, 由式 (2) 集结 3 位专家意 见, 从而构造直觉模糊判断矩阵:

\footnotetext{
$\left[\begin{array}{llllll}(0.500,0.300) & (0.548,0.298) & (0.517,0.315) & (0.700,0.200) & (0.700,0.200) & (0.700,0.200)\end{array}\right]$ $\begin{array}{llllll}(0.469,0.377) & (0.500,0.300) & (0.506,0.321) & (0.700,0.200) & (0.700,0.200) & (0.700,0.200)\end{array}$ $=\begin{array}{llllll}(0.497,0.334) & (0.506,0,321) & (0.500,0.300) & (0.700,0.200) & (0.700,0.200) & (0.700,0.200)\end{array}$ $R=\left(\begin{array}{llllll}(0.300,0.600) & (0.300,0.600) & (0.300,0.600) & (0.500,0.300) & (0.469,0.377) & (0.633,0.234)\end{array}\right.$ $\begin{array}{llllll}(0.300,0.600) & (0.300,0.600) & (0.300,0.600) & (0.548,0.298) & (0.500,0.300) & (0.700,0.200)\end{array}$ $\left[\begin{array}{llllll}(0.300,0.600) & (0.300,0.600) & (0.300,0.600) & (0.327,0.491) & (0.300,0.600) & (0.500,0.300)\end{array}\right]$
}

对于直觉模糊判断矩阵 $R$, 由式（5）和式（6） 计算得到的直觉模糊一致性判断矩阵:

\footnotetext{
$\left[\begin{array}{lllllll}(0.500,0.300) & (0.548,0.298) & (0.554,0.167) & (0.727,0.100) & (0.709,0.109) & (0.779,0.080)\end{array}\right]$ $\begin{array}{llllll}(0.289,0.548) & (0.500,0.300) & (0.506,0.321) & (0.705,0.106) & (0.689,0.118) & (0.789,0.076)\end{array}$ $\bar{R}=\left(\begin{array}{llllll}(0.167,0.554) & (0.321,0,506) & (0.500,0.300) & (0.700,0.200) & (0.673,0.132) & (0.824,0.065)\end{array}\right.$ $\begin{array}{llllll}(0.100,0.727) & (0.106,0.705) & (0.200,0.700) & (0.500,0.300) & (0.469,0.377) & (0.673,0.132)\end{array}$ $\begin{array}{llllll}(0.109,0.709) & (0.118,0.689) & (0.132,0.673) & (0.377,0.469) & (0.500,0.300) & (0.700,0.200)\end{array}$ $\left[\begin{array}{llllll}(0.080,0.779) & (0.076,0.789) & (0.065,0.824) & (0.132,0.673) & (0.200,0.700) & (0.500,0.300)\end{array}\right]$
} 
由式（4）计算得出 $d(\bar{R}, R)=0.197>0.1$, 未通 过一致性检验。设置迭代参数 $\sigma(\sigma \in[0,1])$ 对直 觉模糊一致性判断矩阵 $\bar{R}$ 进行调整。迭代参数 $\sigma$ 从 1 开始以-0.01 为步长, 运用式 (7) 和式 (8) 进行 迭代试验。当 $\sigma=0.41$ 时，由式（9）可得 $d(\tilde{R}, R)=0.098<0.1 ， \tilde{R}$ 通过一致性检验。调整后 的直觉模糊一致性判断矩阵为:

\footnotetext{
$\left[\begin{array}{lllllll}(0.500,0.300) & (0.548,0.298) & (0.532,0.247) & (0.711,0.152) & (0.704,0.157) & (0.734,0.140)\end{array}\right]$ $\begin{array}{llllll}(0.395,0.446) & (0.500,0.300) & (0.506,0.321) & (0.702,0.155) & (0.696,0.162) & (0.739,0.137)\end{array}$ $\widetilde{R}=\left(\begin{array}{llllll}(0.339,0.421) & (0.427,0,393) & (0.500,0.300) & (0.700,0.200) & (0.689,0.169) & (0.756,0.129)\end{array}\right.$ $=\begin{array}{llllll}(0.197,0.655) & (0.202,0.645) & (0.256,0.643) & (0.500,0.300) & (0.469,0.377) & (0.650,0.186)\end{array}$ $\begin{array}{llllll}(0.204,0.647) & (0.210,0.638) & (0.219,0.631) & (0.477,0.364) & (0.500,0.300) & (0.700,0.200)\end{array}$ $\left[\begin{array}{llllll}(0.183,0.681) & (0.179,0.686) & (0.169,0.705) & (0.253,0.568) & (0.256,0.643) & (0.500,0.300)\end{array}\right]$
}

将通过一致性检验的直觉模糊一致性判断矩阵 代入式 (10), 计算可得一级指标的直觉模糊权重:

$\tilde{w}_{1}=(0.166,0.720) ; \quad \tilde{w}_{2}=(0.158,0.733) ;$

$\tilde{w}_{3}=(0.152,0.739) ; \quad \tilde{w}_{4}=(0.101,0.810) ;$

$\tilde{w}_{5}=(0.103,0.808) ; \tilde{w}_{6}=(0.069,0.856)$ 。

同理可得二级指标的直觉模糊权重, 如表 4 所 示。

表 4 二级指标的直觉模糊权重

\begin{tabular}{|c|c|c|c|}
\hline $\begin{array}{c}\text { 二级 } \\
\text { 指标 }\end{array}$ & 直觉模糊权重 & $\begin{array}{c}\text { 二级 } \\
\text { 指标 }\end{array}$ & 直觉模糊权重 \\
\hline$U_{11}$ & $(0.258,0.532)$ & $U_{33}$ & $(0.290,0.504)$ \\
\hline$U_{12}$ & $(0.203,0.618)$ & $U_{41}$ & $(0.340,0.360)$ \\
\hline$U_{13}$ & $(0.282,0.504)$ & $U_{42}$ & $(0.397,0.283)$ \\
\hline$U_{21}$ & $(0.297,0.484)$ & $U_{51}$ & $(0.225,0.574)$ \\
\hline$U_{22}$ & $(0.193,0.641)$ & $U_{52}$ & $(0.259,0.538)$ \\
\hline$U_{23}$ & $(0.257,0.535)$ & $U_{53}$ & $(0.254,0.533)$ \\
\hline$U_{31}$ & $(0.253,0.550)$ & $U_{61}$ & $(0.497,0.209)$ \\
\hline$U_{32}$ & $(0.209,0.618)$ & $U_{62}$ & $(0.275,0.496)$ \\
\hline
\end{tabular}

（3）计算样本企业 $A$ 在第 $i$ 个一级指标下第 $k$ 个 二级指标的直觉模糊评价值

针对样本企业 $A$ 在各一级指标下的每个二级指 标值, 由 3 位专家根据表 3 分别给出指标评价语意变 量。根据专家权重 $(0.3976,0.3012,0.3012)$ ，由式（2） 集结 3 位专家意见, 得出样本企业 $A$ 在第 $i$ 个一级指 标下第 $k$ 个二级指标的直觉模糊评价值 $\tilde{x}_{A i k}$, 如表 5 所示。

（4）计算样本企业 $A$ 在第 $i$ 个一级指标下的直 觉模糊评价值

根据表 5 和表 4, 由式 (11) 计算得出样本企业 $A$ 在第 $i$ 个一级指标下的直觉模糊评价值 $\tilde{x}_{A i}$ :

$$
\begin{array}{ll}
\tilde{x}_{A 1}=(0.281,0.406) ; & \tilde{x}_{A 2}=(0.515,0.213) ; \\
\tilde{x}_{A 3}=(0.520,0.215) ; & \tilde{x}_{A 4}=(0.484,0.186) ; \\
\tilde{x}_{A 5}=(0.496,0.217) ; & \tilde{x}_{A 6}=(0.450,0.224) 。
\end{array}
$$

(5) 计算样本企业 $A$ 的直觉模糊综合评价值

根据一级指标的直觉模糊权重 $\tilde{w}_{i}$ 和样本企业 $A$ 在第 $i$ 个一级指标下的直觉模糊评价值 $\tilde{x}_{A i}$, 由式 （12）计算可得样本企业 $A$ 的直觉模糊综合评价值:

$$
\begin{aligned}
\tilde{x}_{A} & =\tilde{x}_{A 1} \otimes \tilde{w}_{1} \oplus \tilde{x}_{A 2} \otimes \tilde{w}_{2} \oplus \cdots \oplus \tilde{x}_{A 5} \otimes \tilde{w}_{5} \oplus \tilde{x}_{A 6} \otimes \tilde{w}_{6} \\
& =(0.259,0.334) \\
& \text { 同理可得: } \\
& \tilde{x}_{B}=(0.278,0.344) ; \quad \tilde{x}_{C}=(0.225,0.425) 。
\end{aligned}
$$

表 5 样本企业 $A$ 的二级指标直觉模糊评价值

\begin{tabular}{|c|c|c|c|c|}
\hline 二级 & 专家 1 & 专家 2 & 专家 3 & $\begin{array}{c}\text { 直觉模 } \\
\text { 糊评价 } \\
\text { 值 }\end{array}$ \\
\hline \multirow{2}{*}{$U_{11}$} & $(0.35$, & $(0.35$, & $(0.50$, & $(0.399$, \\
& $0.50)$ & $0.50)$ & $0.30)$ & $0.429)$ \\
\hline \multirow{2}{*}{$U_{12}$} & $(0.20$, & $(0.20$, & $(0.35$, & $(0.248$, \\
& $0.75)$ & $0.75)$ & $0.50)$ & $0.664)$ \\
\hline \multirow{2}{*}{$U_{13}$} & $(0.50$, & $(0.50$, & $(0.65$, & $(0.551$, \\
& $0.30)$ & $0.30)$ & $0.20)$ & $0.266)$ \\
\hline \multirow{2}{*}{$U_{21}$} & $(0.80$, & $(0.80$, & $(0.95$, & $(0.868$, \\
& $0.15)$ & $0.15)$ & $0.05)$ & $0.108)$ \\
\hline \multirow{2}{*}{$U_{22}$} & $(0.65$, & $(0.65$, & $(0.80$, & $(0.704$, \\
& $0.20)$ & $0.20)$ & $0.15)$ & $0.183)$ \\
\hline \multirow{2}{*}{$U_{23}$} & $(0.95$, & $(0.95$, & $(0.95$, & $(0.950$, \\
& $0.05)$ & $0.05)$ & $0.05)$ & $0.050)$ \\
\hline \multirow{2}{*}{$U_{31}$} & $(0.65$, & $(0.65$, & $(0.80$, & $(0.704$, \\
& $0.20)$ & $0.20)$ & $0.15)$ & $0.183)$ \\
\hline \multirow{2}{*}{$U_{32}$} & $(0.95$, & $(0.95$, & $(0.80$, & $(0.924$, \\
& $0.05)$ & $0.05)$ & $0.15)$ & $0.070)$ \\
\hline \multirow{2}{*}{$U_{33}$} & $(0.95$, & $(0.95$, & $(0.95$, & $(0.950$, \\
& $0.05)$ & $0.05)$ & $0.05)$ & $0.050)$ \\
\hline \multirow{2}{*}{$U_{41}$} & $(0.80$, & $(0.80$, & $(0.65$, & $(0.763$, \\
& $0.15)$ & $0.15)$ & $0.20)$ & $0.164)$ \\
\hline \multirow{2}{*}{$U_{42}$} & $(0.80$, & $(0.80$, & $(0.65$, & $(0.763$, \\
& $0.15)$ & $0.15)$ & $0.20)$ & $0.164)$ \\
\hline \multirow{2}{*}{$U_{51}$} & $(0.50$, & $(0.65$, & $(0.65$, & $(0.596$, \\
& $0.30)$ & $0.20)$ & $0.20)$ & $0.235)$ \\
\hline \multirow{2}{*}{$U_{52}$} & $(0.95$, & $(0.95$, & $(0.80$, & $(0.924$, \\
& $0.05)$ & $0.05)$ & $0.15)$ & $0.070)$ \\
\hline \multirow{2}{*}{$U_{53}$} & $(0.95$, & $(0.95$, & $(0.80$, & $(0.924$, \\
& $0.05)$ & $0.05)$ & $0.15)$ & $0.070)$ \\
\hline \multirow{2}{*}{$U_{61}$} & $(0.65$, & $(0.65$, & $(0.50$, & $(0.610$, \\
& $0.20)$ & $0.20)$ & $0.30)$ & $0.226)$ \\
\hline \multirow{2}{*}{$\begin{array}{c}(0.80, \\
0.15)\end{array}$} & $(0.80$, & $(0.65$, & $(0.763$, \\
& & & $0.20)$ & $0.164)$ \\
\hline
\end{tabular}

（6）计算样本企业的信用综合记分

由式 (13) 计算可得样本企业 $A$ 的信用综合记 分:

$$
S\left(\tilde{x}_{A}\right)=0.259-0.334+\frac{1.221-1}{1.221+1} \times 0.371=-0.002
$$

其 中, $\operatorname{shi}\left(\tilde{x}_{A}\right)=0.883 ; \operatorname{shi}\left(\tilde{x}_{B}\right)=0.808$; $\operatorname{shi}\left(\tilde{x}_{C}\right)=0.529 ; \quad \operatorname{shi}(I)=0.723 ; \theta_{A}=1.221 ;$ $\theta_{B}=1.117 ; \theta_{C}=0.732$ 。

同理可得:

$S\left(\tilde{x}_{B}\right)=-0.045 ; \quad S\left(\tilde{x}_{C}\right)=-0.254$ 。

为更直观地反映样本企业的信用状况, 本文对 样本企业的信用综合记分进行百分制标准化处理, 
即有

$$
\begin{aligned}
& S^{\prime}\left(\tilde{x}_{A}\right)=\frac{(-0.002)-(-1)}{1-(-1)} \times 100=49.90 ; \\
& S^{\prime}\left(\tilde{x}_{B}\right)=47.75 ; \quad S^{\prime}\left(\tilde{x}_{C}\right)=37.30 。
\end{aligned}
$$

由此可见，样本企业的信用综合记分排序为: $S^{\prime}\left(\tilde{x}_{A}\right) \succ S^{\prime}\left(\tilde{x}_{B}\right) \succ S^{\prime}\left(\tilde{x}_{C}\right)$, 表明样本企业 $A$ 的信用风 险相对较低, 样本企业 $C$ 的信用风险相对较高。

据企业信用网（http://www.bgcheck.cn/\#\#\#\#）显 示: 网宿科技的信用等级为 $\mathrm{A}$ 级、立思辰的信用等 级为 $\mathrm{BBB}$ 级、天喻信息的信用等级为 $\mathrm{BB}$ 级, 本文 模型得出的排序结果与企业信用网完全一致，证明 了本文模型的可行性和有效性。

为便于比较, 本文还运用模糊综合评价法对样 本企业的信用状况进行了评价。其中, 备择集设定 为: $V=\{$ 好, 较好, 一般, 较差, 很差 $\}$; 利用正态 隶属函数确定指标的隶属度; 采用 IFE-熵权法 ${ }^{[16]}$ 确 定指标权重。根据最大隶属度原则, 样本企业 $A$ 的 语言评价值为一般; 样本企业 $B$ 的语言评价值为较 差; 样本企业 $C$ 的语言评价值为很差。由此可知, 模糊综合评价法得出的排序结果与本文模型一致, 但模糊综合评价法只能给出样本企业信用状况的语 言评价值, 而本文模型既能给出直觉模糊综合评价 值, 又能给出精确的信用综合记分。

\section{6. 结束语}

本文针对传统模糊集理论不能准确反映决策者 犹豫不决或弃权情况的问题, 引入直觉模糊集, 构 建基于直觉模糊集的大数据企业信用风险评价模型。 其中, 采用直觉模糊层次分析法确定各级指标的直 觉模糊权重; 根据直觉模糊数的运算法则, 构造直 觉模糊加权平均算子 (IFWA算子), 并计算待评企 业的直觉模糊综合评价值; 利用直觉模糊数记分函 数, 计算得出待评企业的信用综合记分和排序。以 大数据产业上市公司为样本, 实例分析结果证明了 该模型的可行性和有效性。该模型能够较好、较完 整地反映专家对大数据企业信用风险评价的模糊性 和不确定性, 为大数据企业信用风险评价提供了一 种新的方法。在实际应用中, 专家给出的隶属度、 非隶属度和犹豫度应是一个区间值, 如何在本文模 型中引入区间直觉模糊数值得进一步研究。

\section{致谢}

This research was funded by the Regional Project of National Natural Science Foundation of China, grant number 71861003 .

\section{参考文献}

[1] 工业和信息化部.大数据产业发展规划(2016-2020 年 )( 工 信 部 规 〔2016〕 412 号)[EB/OL]. http://www.miit.gov.cn,2017-1-17.
[2] 国务院. 促进大数据发展行动纲要(国发 (2015) 50 号)[EB/OL]. http://www.gov.cn,2015-9-5.

[3] 魏巍贤.企业信用等级综合评价方法及应用 [J].系 统工程理论与实践,1998,18(2):26-31.

[4] 陈晓红,杨志慧. 基于改进模糊综合评价法的信用 评估体系研究一以我国中小上市公司为样本的 实证研究[J].中国管理科学,2015,23(1):146-153.

[5] Atanassov K T. Intuitionistic Fuzzy Sets[J]. Fuzzy Sets and Systems, 1986,20(1):87-96.

[6] Zadeh L A. Fuzzy Sets[J]. Information and Control,1965,8(3):338-353.

[7] 谭春桥.基于区间值直觉模糊集的 TOPSIS 多属性 决策 $[J]$.模糊系统与数学,2010,24(1):92-97.

[8] 王开荣,杨红.基于直觉模糊集的多属性决策方法 [J].模糊系统与数学, 2010,24(3):114-118.

[9] 许进,陶克涛.科技型中小企业信用评估的指标体 系设计 [J].科学管理研究,2006,24(3):55-58.

[10] Xu Z S. Intuitionistic Fuzzy Aggregation Operators[J]. IEEE Transactions on Fuzzy Systems,2007,15 (6):1179-1187.

[11] 李鹏,刘思峰,朱建军.基于前景理论的随机直 觉模糊决策方法 [J].控制与决策,2012,27(11):16011606.

[12] 李艳红.基于模糊 AHP 考虑专家权重的战略 性新兴产业评价与选择——唐山市的实证分析 $[\mathrm{J}]$. 中国集体经济,2017,(6):60-62.

[13] 高红云,王超, 哈明虎.直觉模糊层次分析法 [J]. 河北工程大学学报(自然科学版),2011,28(4):101105.

[14] Szmidt E, Kacprzyk J. Amount of Information and its Reliability in the Ranking of Atanassov's Intuitionistic Fuzzy Alternatives[A]. Rakus-Adersson E, Yager R R, Ichalkaranjie N, et al. Recent Advances in Decision Making[M]. Berlin: Springer,2009:7-19.

[15] Xu Z S, Liao H C. Intuitionistic Fuzzy Analytic Hierarchy Process[J]. IEEE Transactions on Fuzzy Systems, 2014,22(4):749-761.

[16] 孟俊娜,房宁,刘炳胜,薛斌.基于直觉模糊集的 城镇基础设施项目可持续性评价 [J]. 模糊系统与 数学, $2015,29(1): 167-174$. 